\title{
A STUDY OF VARIOUS VIEWPOINTS AND ASPECTS : SOFTWARE QUALITY PERSPECTIVE
}

\author{
Jogannagari Malla Reddy ${ }^{1}$, S.V.A.V. Prasad ${ }^{2}$ \\ ${ }^{1}$ Research Scholar, Dept. of Computer Science \& Engineering, Lingaya's University, Faridabad, Haryana, India \\ ${ }^{2}$ Professor \& Dean( Research \& Development), Lingaya's University, Faridabad, Haryana, India
}

\begin{abstract}
The software quality is very important research of software engineering grown from the last two decades. The software engineering paradigm adopted by many organizations to develop the high quality software at affordable cost. The high quality software is considered as one of the key factor in the rapid growth of Global Software Development. The software metrics computes and evaluates the quality characteristics and used to take quantitative and qualitative decisions for risk assessment and reduction. The multiple stakeholders can view the software quality in multiple angles with various aspects. In this paper we present multiple views of the software quality with respect to various quality aspects.
\end{abstract}

Key Words : Stakeholders, Functional aspect, Structural aspect, Process aspect, Metrics etc.

\section{INTRODUCTION}

The software engineering discipline that focus on the cost effective development of high quality software. The software is logical product and intangible The software organizations are commitment to quality. Many software organizations have been seriously concentrating on effective ways to improve the product quality. The quality culture ultimately leads to development of more effective approaches in the software engineering. As many companies in the software industry still not following software engineering approaches effectively in their software development projects still produces software with time delay, over budget and not realistic. The delivered software products should meets the customer expectations. The lack of quality has leads to significant costs in terms of money and time to the developers and unsatisfaction to the users. The faulty systems can't fulfill the goals of customers.

The following effect is on the internal and external of organization over the time due lack of software quality.

- Economical loss due to lost business.

- Economical loss for payment of compensations

- Financial loss occurred with lost customers.

- Unnecessary investment can be made due to legal consequences and suits.

- The damage of firm brand image.

The software engineering provides a rich set of frame works, methods and tools in aid of development of software projects. The software product quality is highly related to the quality of the software process The software process consists the dominant feature of framework activity for planning, conceptualizing, designing ,construction and deployment. A software standards recommends the mandatory methods, rules, requirements and practices to be employed during the software development. The standards make possible to measure the size, content, value or quality of software entity. Measurement is the process that helps the organizations to evaluate their products. Software metrics measure different aspects of software complexity, which play the crucial role in analyzing and enhance the software quality.

This paper presents various aspects of software quality with respect to the different stakeholders.. The organization of the rest of the paper is as follows. The Section 2 describes the related work focusing prior work on quality metrics. The Section 3 states the General definitions, Viewpoints and Aspects of the software quality. Section 4 Discussion of metrics for improving the software quality. Finally a discussion about Conclusions and future scope is given in the Section 5.

\section{RELATED WORK}

Over the years eminent theorists defined the quality with various concepts. These concept taxonomy can be benefit for discussion, setting up and evaluating the software products. Apart from that, researchers designed and implemented different software quality assurance techniques and models for enhancement of quality culture. The number of researchers have worked to address various issues in this domain of software quality metrics.

- H.F. LI derived the empirical study on various metrics using a Fortran Static source code analyzer including with new hybrid metrics. The hybrid metric can incorporate both volume and control attributes in assessing software product complexity. 
- Sadia Rehman et al[2] described the role of software metrics in the global software development with systematic literature for data search.

- Dr. Deepshikha Jamwal [3] compared the different software quality models with each other. Some recommendation made for future researchers.

- Mrinal Singh Rawat et al[4] focuses on the software quality with extendable knowledge on multiple stakeholders of the software product. The author compared of software metrics with strengths and weaknesses.

- Kitchenham [5] defined the quality as complex, it is highly context dependent. The diffe rent stakeholders are relevant with the quality. There is no single, simple measure for measuring the software quality which is acceptable by everyone.

- Shyam.R et al[6] addresses the needs through the development and implementation of new suite of metrics for OO design. The research paper contributed the development and empirical validation of a set of theoretical ground of metrics on object oriented design.

- ISO quality standards adapted by organizations to focus the their performance levels. The various internal and external quality factors are focused in the ISO 9126 quality model.

- David Chappell presented the paper on software quality in stakeholder point of view. Chappell considered the process quality as more important.

- Gurdev Singh et al[7] presented the research paper on classification of software metrics. with advantages and limitations.

- Munch[8] proposed context-oriented alignment of process patterns, project goals and characteristics of project environment. The paper presented with an abstract of one or more software develop ment processes.

- Barbara Kitchenham has held a survey on advancement of software metrics research with 103 papers published in between 2000 and 2005. She suggested the researchers need to refine the empirical methodology to solve the software quality metric problems.

- According to the Tom Demcrio "You need not control what you can't measure". The quality measurement is prerequisite to management control in the organizations [9]

- Rafa E. Al-Qutaish[10] assured the software product quality during the software development life cycle with ISO perceptive. The author derived the weakness of the cross references between the two ISO standards had been highlighted.
- Mbusi Sibisi et al[11] developed a process framework that can be used to customize or tailor software quality models to fulfill the needs of customer and organization.

- A Review on Software Quality Models presented by $\mathrm{Mr}$ Brijendra Singh et al[12] with quality attribute comparison based on Software quality process and software quality models.

- Tharashasank Davuluru et al[13] compared the characteristics with software quality models and proposed future frame work for modeling the quality of web based systems.

- According to Gordon[14], software quality is important characteristic affecting overall system development lifecycle cost and performance.

\section{GENERAL DEFINITIONS, VIEWPOINTS AND ASPECTS OF SOFTWARE QUALTIY}

Software quality is an important issue in the development of successful product. Quality of software is often expressed in its internal and external attributes which are subdivided into the other attributes such as flexibility, efficiency, maintainability, testability, interoperability, portability, and reusability. A metric is quantifiable measurement of software product, or project that directly observed and calculated. Software metrics provide a quantitative basis for planning and predicting software development processes. Many metrics and models have been developed, promoted and utilized resulting in remarkable successes.

In this section, we would first look the basic definitions of Software Metrics defined by eminent persons and reputed organizations followed by an overview of metrics.

\subsection{Definitions}

- "Software Quality is the degree to which a system, component, or process meets specified requirements and meets customer needs or expectations". [IEEE ]

- "Quality comprises all characteristics and significant features of a product or activity which relate to the satisfying of given require ments". [German Industry Standard DIN 55350 Part 11].

- "Quality is the totality of features and characteristics of a product or a service that bears on its ability to satisfy the give needs". [ANSI Standard 1978 ]

- "The continuation application of measurement-based techniques to the software development process and its products to supply meaningful and timely management information, together with the use of those techniques to improve the process and its products". [ Paul Goodman] 
- "You cannot control what you cannot measure". [Tom Demarco]

- " I know no way of judging the future, but by the past". [Patrick's Henry]

- The definitions provides more scope to discuss the software quality on various aspects in multiple views.

\subsection{The Viewpoints of the Software Quality}

The project stakeholder is an individual actively involved in a project, or whose interest might in direct or indirect as of result of project execution.

The influential research paper theorized by David \& Garvin states that " software Quality depends on number of factors which is perceived in various domains, including economics, philosophy, marketing and operational research". He concluded the concept "quality is complex and multifaceted " that can be described in different views which are follows .

- The user view sees the quality as fitness for operational use.

- The Manufacturing view sees quality as conformance to specification.

- The product view looks the quality is tie-up internal features of the product.

- The Value-based view sees the quality is relevant to the cost to benefit angle.

The different views can be held by different groups involved in the software development. Customers and marketing groups typically in the user view, researchers and innovators are in product view, the production people, development teams are in manufacturing view and product purchasers take rather different value-based view.

User view : The product usability is related with user view. This software quality is high subjective depending on product characteristics that meets the user's needs. The quality evaluates the product as highly personalized view. The product should be more flexible to operate, usable in their environments that is expected to functionality and usage pattern like business, laboratories etc.

Manufacturing View: The model ISO 9001 and the Capability Maturity Model focuses on manufacturing view that stress on following process as opposed to going by specification. The manufacturing view encompasses the product quality during production and after delivery. The best and high quality manufacturing process will automatically lead to a better product, that cannot be inferred with substandard product. The conformance to process standards guarantees good products. The both models ISO as well as the CMM , indirectly do imply that the concept of " Documenting what you do and doing what you say" helps in improving the product quality.
Product View : The product viewpoint focuses at the inherent features as well as the characteristics of the product. This approach is frequently adopted by softwaremetrics. The idea of measuring internal product properties( internal quality indicators ) leads to improve the external product behaviour. There is broad scope in development of models to link the product view to the user view.

Value based view : The value-based view becomes important when there are lot of contrasting views in diffe rent departments of the organization. The customers generally take a user view and the technical department will generally take a product view. Though initially these different viewpoints helps to develop the software product with 360 degrees in quality with different viewpoints. The user's requirement for product may in conflict with manufacturer's goal of minimizing rework. A value based view technique can mange the conflicts when requirements are changed. The view resolve such issues by looking at the issue in relation in terms of cost, constraints, time and resources. The value based viewpoint looks the conflict with a cost to benefit angle.

\subsection{The Aspects of the Software Quality}

The group of people i.e. users, developers and management sponsors interested in software quality and they can face outcome of defects in externally or internally over the time. The stakeholders may care about the quality in different aspects like functional, structural and process aspect. Each quality aspect being relevant in some way to these group of people. The users are interested in functional aspect and little bit interest on the delivery schedule of process aspect. Developers have much interest on the structural aspect (i.e code), but also have less interest on functional aspect than the users. The project development process to meet a deadline by improving process quality. The management sponsors are indirect users of the product they relevant with all aspects of the software quality. The change in one aspect leads the impact on other aspect as positive or negative.

Functional Aspect : The functional aspect fulfill the functions requirements of the system/ user. The functional aspect is the service the system should provide, how the system should react in particulars inputs and how the system should behave in particular situations and conditions (i.e input, output, exceptions). Some times that may also explicitly state what the system should not do. Failure to meet an individual functional aspect requirements may leads to degraded system. The attributes are following..

Meeting the functional requirements: These requirements are services collected from the management sponsors and intended users of the project. The functional quality should meet the requirements in consistent and complete. The dynamic learning systems are practically impossible 
to achieve requirement with consistence and completeness.

Product with few defects. : The software with bugs can reduce the reliability, compromise the security. The system should correctly deliver the services as expected by the user over the period of time.

Product with good performance : From the user's point of view, there's no such software product as a good when it slow performance.

Performance of User Interface design : the functional quality depends on the product user interface. The aesthetic elements, navigational elements and technical elements can in increase the performance and make more beautiful of user interface design.

Structural Aspect : The structural aspect is relevant with the software code. The structured programming can reduce code complexity and increases the structural quality of the product. The various tools used to improve and measure the software structural quality. The structural quality depends on the following attributes.

* Testability : The source code organized in the way of testable.

* Maintainability: The source code is to modifiable according to operational \& technical change.

* Understandability: The code is unambiguous, easy to readable and understandable to new developers.

* Efficiency: The efficiency is resource-constrained. The efficient programming can be critically important.

* Security : The software should prevent common attacks and malfunctioning of unauthorized.

Process Aspect : The process aspect critically important to the users, developers and management sponsors. The three stake holders are interested the following attributes in getting project deadline .

Time constraint: The software should delivered with in time schedule or not. ?

Budget constraint: The software should delivered with in budget or not.?

Operational constraint: The software should possible to executable in environment or not?

\section{METRICS FOR IMPROVING THE QUALITY}

The software is not only coding of program, it is complex process with engineering vision, analytical thinking, modeling and software process management supported tools. The quality assurance is crucial in software development.
Quality standards will also be used for upcoming projects and increase company growth. Testing is very important for software quality. Tools for improving functional quality through manual testing tools through user interface and automated testing tool, such as frameworks for unit testing. The software refactoring improves the structural quality of the product. The developer improve the code structure without changing it behavior. The structural quality tools encompasses the static code analysis, examining code for security attacks and measure the test coverage. These tools provide the code metrics and measure the cyclomatic complexity. The process metrics improve the process quality of the development process. The tools will track the process status, tracing the progress against requirements and measure the project fitness.

\section{CONCLUSION AND DISCUSSION}

The software quality is not possible to view in single direction. The quality visualize based on various perspectives of the stakeholders. The three areas of aspects help us to think about the kinds of tools for improving the software quality. A various metrics are exercised for measuring the software product quality prior to implementation. Future research directions include improvement in existing metrics based on nature of the aspect and magnitude of the problem statement.

\section{ACKNOWLEDGEMENT}

We sincerely acknowledge to all the academicians of the Department of Computer Science \& Engineering, Lingaya's University, Faridabad for their motivation, support and cooperation during the study.

\section{REFERENCES}

[1] H.F.LI , “An Emp irical Study of Metrics”, IEEE Transaction on Software Engineering, Vol. SE-13, No. 6, June, 1987

[2] Sadia Rehman et al, "Swot Analysis of Software Quality Metrics for Global Soft ware Development : A Systematic Literature Review Protocol", IOSR Journal of Computer Engineering, Vol.2. Issue 1, 2012

[3] Dr. Deepshikha Jamwal, “ Analysis of Software Quality Models for Organizations", International Journal of Latest Trends in Computing, Vol. 1, Is sue. 2, Dec 2010

[4] Mrinal Singh Rawat et al, " Survey on Impact of Software Metrics on Software Quality ", International Journal of Advanced Computer Science \& Applications, Vol. 3., No. 1, 2012

[5] B. Kitchenham, "What's up with software metrics?- A Preliminary mapping study", The Journal of Systems and software", 2010

[6] Shyam R. et al, "A Metrics Suite for Object Oriented Design", IEEE Transactions on Software Engineering, Vol. 20, No. 6, June 1994.

[7] Gurdev Singh et al, " A Study of Software 
Metrics", International Journal of Computational Engineering \& Management, Vol. 11, Jan, 2011.

[8] Munch j, “ Goal-oriented Composition of

Software Process Patterns", Proc. of the $6^{\text {th }}$ International Workshop on Software Process

Simulation and Modeling, pp. 164-168, 2005.

[9] Barbara Kitchenham et al, " Software Quality : The Exclusive Target”, IEEE publication, Jan, 1966.

10] Rafa E. Al-Qutaish, "Measuring the Software Product Quality during the Software Development Life Cyle - An ISO Standards Perspective, Journal of Computer Science.5(5) , pp 392-397, 2009.

[11] Mbusi Sibisi et al, " A Process Framework for Customizing Software Quality Models ”, IEEE Manuscript, Mar 2007.

[12] Brijendra Singh et al, "A Review on Software Quality Models", International Conference on Communication Systems and Network Technologies.

[13] Tharashasank Davuluru et al, " A Study of Software Quality Models”, IEEE - ICAETR2014, Unnao, India, Aug 2014.

[14] Gordon W. Skelton, "Integrating total quality management with software engineering education”, ACM Vol 25 Issue 2, June 1993

\section{BIOGRAPHIES}

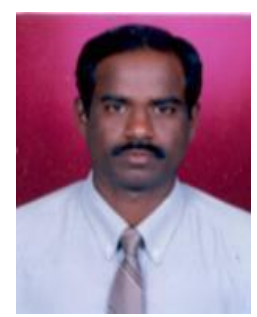

Jogannagari Malla Reddy obtained M.Tech (CSE) from JNTU, Hyderabad. and pursuing $\mathrm{Ph} . \mathrm{D}$ (CSE) from Lingaya's Uni versity, Faridabad. His area of specialization in software Engineering, Management Information Systems and Database Management Systems. He published various papers on Information Systems in various National and International reputed Journals \& conferences.

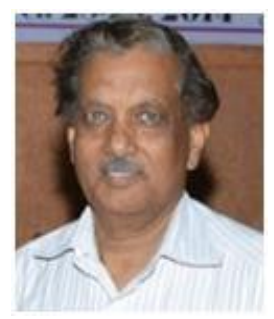

Dr. S.V.A.V. Prasad done his PhD from Andhra University, presently he working as Professor \& Dearn (R\&D) in Lingaya's University, Faridabad, has 30 years of experience in Teaching and R\&D. He published various research papers in National and International reputed Journals \& Conferences. He guided many students in research progrmmes at university in communication system and information system areas . 